\title{
Prevalência e Fatores Associados à Polifarmácia em Idosos Atendidos na Atenção Primária à Saúde em Belo Horizonte-MG, Brasil
}

\author{
Prevalence and factors associated with polypharmacy among the \\ elderly treated in Primary Healthcare in Belo Horizonte, State of \\ Minas Gerais, Brazil
}

Patrícia Carvalho de Oliveira (https://orcid.org/0000-0001-9519-719X) ${ }^{1}$

Micheline Rosa Silveira (https://orcid.org/0000-0001-7002-4428) ${ }^{2}$

Maria das Graças Braga Ceccato (https://orcid.org/0000-0002-4340-0659) ${ }^{2}$

Adriano Max Moreira Reis (https://orcid.org/0000-0002-0017-7338) ${ }^{2}$

Isabela Vaz Leite Pinto (https://orcid.org/0000-0001-6335-5480) ${ }^{1}$

Edna Afonso Reis (https://orcid.org/0000-0003-1465-9167) ${ }^{1}$
${ }^{1}$ Departamento de Estatística, Instituto de Ciências Exatas, UFMG Av. Antônio Carlos 6627/ ICEx/DEST/s4054, Pampulha. 31270-901 Belo Horizonte MG Brasil. ednareis@gmail.com ${ }^{2}$ Faculdade de Farmácia, UFMG. Belo Horizonte MG Brasil.

\begin{abstract}
The scope of this article is to analyze the prevalence of polymedication and excessive polypharmacy, as well as associated factors, among the elderly attended at two Basic Health Units in Belo Horizonte, State of Minas Gerais. A cross-sectional observational study was conducted using information obtained from a structured interview of the patient. The individual associations of each explanatory variable with polypharmacy and with excessive polypharmacy were analyzed. For the variables that showed a significant association with polypharmacy, multivariate analysis was performed using the logistic regression model. The elderly used, on average, 5.2 drugs. The prevalence of polymedication was $57.7 \%$ and excessive polypharmacy was $4.8 \%$. In univariate analysis the conditions associated with polypharmacy were: age $\leq 70$ years, schooling $>$ 8 years, presence of more than three diseases and presenting symptoms of depression. For excessive polypharmacy, the presence of more than three diseases, self-perception of negative health, and partial dependence on instrumental activities of daily living were associated with the conditions. In the final multivariate model for polypharmacy, the age $\leq 70$ years and presence of more than three disease variables remained.
\end{abstract}

Key words Polymedication, Elderly, Drug therapy, Pharmaceutical care, Primary healthcare
Resumo $O$ objetivo deste artigo é analisar a prevalência de polifarmácia e de polifarmácia excessiva, bem como seus fatores associados, entre idosos atendidos em duas Unidades Básicas de Saúde de Belo Horizonte-MG. Foi conduzido um estudo observacional transversal, realizado por meio de informações resultantes de uma entrevista estruturada ao paciente. Foram analisadas as associações individuais de cada variável explicativa com a polifarmácia e polifarmácia excessiva. Para as variáveis que apresentaram associação significativa com polifarmácia, foi realizada análise multivariada por meio do modelo de regressão logística. Os idosos utilizavam, em média, 5,2 fármacos. A prevalência de polifarmácia foi de $57,7 \%$ e de polifarmácia excessiva foi de $4,8 \%$. $\mathrm{Na}$ análise univariada, mostraram-se associadas à polifarmácia as condições idade $\leq 70$ anos, escolaridade $>8$ anos, presença de mais de três doenças e presença de sintomas de depressão. Para polifarmácia excessiva, mostraram-se associadas as condições presença de mais de três doenças, autopercepção da saúde negativa e dependência parcial nas atividades instrumentais de vida diária. No modelo multivariado final para polifarmácia, permaneceram as variáveis idade $\leq 70$ anos e presença de mais de três doenças.

Palavras-chave Polimedicação, Idoso, Tratamento farmacológico, Assistência farmacêutica, Atenção Primária à Saúde 


\section{Introdução}

O envelhecimento progressivo da população se deve ao aumento da expectativa de vida por melhoria das condições de saúde, bem como à redução da taxa de fecundidade observada nos últimos anos ${ }^{1}$. Esse cenário contribui para o aumento do contingente de pessoas que desenvolvem condições crônicas de saúde, o que dificulta o manejo terapêutico para os profissionais de saúde e pacientes, comprometendo os resultados de saúde ${ }^{2}$. Além disso, os pacientes que vivem com multimorbidade (presença concomitante de duas ou mais condições crônicas de saúde), demandam, em geral, assistência contínua e a utilização de vários medicamentos ${ }^{3-5}$.

A polifarmácia, que usualmente se refere ao uso concomitante de vários medicamentos, é comum entre idosos com multimorbidade ${ }^{2,6,7} \mathrm{e}$ está associada a desfechos negativos em saúde, como eventos adversos a medicamentos, quedas, fraturas, hospitalizações, aumento do tempo de permanência no hospital, readmissão ao hospital logo após a alta e óbito². Os eventos adversos a medicamentos, que podem ocorrer tanto por reações adversas quanto por interações medicamentosas, têm a polifarmácia como protagonista, uma vez que, quanto maior é o número de medicamentos utilizados, maior é o risco de eventos adversos ${ }^{2,8}$. Esse risco é maior entre pacientes idosos, devido a diversas alterações decorrentes da idade, como diminuição da função renal e hepática, redução da audição, visão, cognição e mobilidade, além de menor massa corporal magra ${ }^{2}$. Portanto, esses pacientes são mais vulneráveis a alterações farmacocinéticas e farmacodinâmicas, estando mais sensíveis à farmacoterapiå.

Na literatura, são empregadas diversas definições para polifarmácia, sendo considerada como uma simples contagem numérica isolada ou associada ao tempo de duração de um determinado tratamento ou à adequação a situação clínica do indivíduo ${ }^{2}$. Embora o termo polifarmácia definido como "o uso de cinco ou mais medicamentos" seja o mais relatado nos estudos ${ }^{2,9,10}$, existem diversas definições numéricas de polifarmácia, variando desde "dois ou mais" até "11 ou mais" medicamentos. Alguns estudos empregaram, ainda, o termo "polifarmácia excessiva", definido como "o uso concomitante de dez ou mais medicamentos"10-13. Logo, não há uma definição padrão usada de forma consistente e universalmente aceita, o que dificulta a avaliação da efetividade e segurança de um determinado tratamento por parte dos profissionais de saúde ${ }^{2,14}$.
Diante do exposto, no presente estudo tem-se como objetivo analisar a prevalência de polifarmácia e de polifarmácia excessiva, e identificar seus fatores associados, entre idosos usuários de medicamentos atendidos em duas Unidades Básicas de Saúde (UBS) de Belo Horizonte, Minas Gerais, no período de novembro de 2013 a abril de 2014.

\section{Métodos}

\section{Desenho do estudo e entrevistas}

Trata-se de estudo observacional transversal, integrante do estudo "Avaliação do acesso a medicamentos e da compreensão do tratamento medicamentoso por idosos atendidos em Unidades Básicas de Saúde em Belo Horizonte - MG", desenvolvido em duas UBS localizadas no Distrito Sanitário Leste do Município de Belo Horizonte, no período de novembro de 2013 a abril de $2014^{15}$. O Distrito Sanitário Leste é o segundo distrito de Belo Horizonte com maior percentual de idosos em sua população $(17,1 \%, n=34.368)$. As duas UBS selecionadas apresentam percentuais significativos de idosos nas suas populações, correspondendo a cerca de $20 \%$ em cada uma ${ }^{16}$.

Para o cálculo do tamanho da amostra, foram empregadas informações presentes no sistema informatizado de gestão da Secretaria Municipal de Saúde de Belo Horizonte. Tal sistema permite o acompanhamento da dispensação de medicamentos de forma online e integrada entre as unidades, permitindo melhor gerenciamento do serviço e disponibilização de informações que auxiliam no acompanhamento da utilização do medicamento pelo usuário ${ }^{17}$. Obteve-se o número mensal médio (janeiro a outubro de 2013) de 483 idosos atendidos pelas farmácias das duas UBS selecionadas. Tendo em vista que os atendimentos são mensais, considerou-se que esses seriam os mesmos idosos que compareciam às farmácias uma vez ao mês para adquirir os medicamentos de uso crônico. Dessa forma, o tamanho da população de estudo foi definido como $\mathrm{n}=483$. Adotando-se uma prevalência de $50 \%$ para todas as características estudadas, margem de erro de $5 \%$ e nível de confiança de $95 \%$, obteve-se tamanho de amostra de 215 idosos, calculado por meio do programa Open Epi versão $3.01^{18}$. Considerando possíveis perdas por recusa à participação na pesquisa, o tamanho final da amostra foi definido em 227 idosos.

Foram incluídos no estudo idosos com idade igual ou maior que 60 anos, que receberam, no 
momento da entrevista, pelo menos um medicamento nas farmácias das duas UBS, e que aceitaram participar da pesquisa, assinando do Termo de Consentimento Livre e Esclarecido. Foram excluídos os idosos com impossibilidade de comunicação oral e/ou visual, bem como aqueles que não estavam utilizando medicamentos no momento da entrevista. O estudo foi previamente aprovado no Comitê de Ética em Pesquisa da Universidade Federal de Minas Gerais e da Secretaria Municipal de Saúde de Belo Horizonte.

Os idosos foram entrevistados por meio de um questionário estruturado contendo perguntas sobre características sociodemográficas, clínicas, funcionais e relacionadas à utilização de medicamentos. Para os idosos que utilizavam medicamentos com o auxílio de outra pessoa, a mesma era entrevistada conjuntamente ao participante. A amostra foi selecionada de forma gradual; após o atendimento nas farmácias, cada idoso era convidado a participar do estudo.

\section{Variáveis e análise estatística}

As variáveis resposta foram polifarmácia, definida pelo uso de cinco ou mais medicamentos ( $\mathrm{sim}$ ou não), e polifarmácia excessiva, definida como o uso de dez ou mais medicamentos (sim ou não).

As variáveis explicativas investigadas foram de natureza: sociodemográfica (sexo, idade, cor da pele, escolaridade, estado civil, morar sozinho(a) e renda); clínica (número de doenças, autopercepção de saúde e sintomas de depressão); funcional (cognição, atividades básicas e instrumentais da vida diária); e relacionada à utilização de medicamentos (classe terapêutica utilizada e adesão ao tratamento).

Cada variável sociodemográfica foi redefinida em duas categorias: o estado civil em "tem companheiro" (casado ou união estável) e "não tem companheiro" (solteiro, viúvo, separado ou divorciado); a idade em " $\leq 70$ anos" e " $>70$ anos"; a cor da pele em "branca ou amarela" e "preta, parda ou indígena"; a escolaridade em " $\leq 8$ anos" e " $>8$ anos"; e a renda em " $\leq 3$ salários mínimos" o " $>3$ salários mínimos".

A variável número de doenças foi classificada em $\leq 3$ ou $>3$ (multimorbidade). Apesar de Harrison et al. ${ }^{19}$ definir multimorbidade como a presença de duas ou mais doenças, no presente estudo escolheu-se elevar o ponto de corte pelo fato do estudo incluir idosos que receberam pelo menos um medicamento na farmácia no momento da entrevista, ou seja, que já apresentavam pelo menos uma doença.
A autopercepção da saúde foi utilizada com a finalidade de conhecer a satisfação do indivíduo com sua saúde. Consiste na utilização de uma questão retirada do Instrumento de Avaliação da Qualidade de Vida "WHOQOL-bref" ${ }^{20}$, desenvolvido em 1998 pelo Grupo de Qualidade de Vida da Organização Mundial de Saúde (OMS) e aplicado no Brasil por Fleck et al ${ }^{21}$. Esta questão apresenta cinco opções de resposta, que foram dicotomizadas como "positiva" (excelente, muito boa ou boa) e "negativa" (regular ou ruim).

A Escala de Depressão Geriátrica de 15 itens (GDS-15) de Yesavage et al. ${ }^{22}$, traduzida para o português por Almeida e Almeida ${ }^{23}$ e validada para o ambiente ambulatorial por Paradela et al. ${ }^{24}$, foi utilizada para avaliar possível quadro de sintomas de depressão em idosos. Nessa escala, considerou-se como tendo sintomas de depressão os idosos que apresentaram $\geq 6$ pontos, o que definiu a categoria "sim" (com sintomas de depressão); portanto, aqueles indivíduos que apresentaram $<6$ pontos foram inseridos na categoria "não" (sem sintomas de depressão).

O Mini Exame do Estado Mental, proposto por Folstein et al. ${ }^{25}$ e adaptado para o Brasil por Bertolucci et al. ${ }^{26}$ e Lourenço e Veras ${ }^{27}$, foi empregado para avaliar a cognição do idoso. A classificação é realizada de acordo com a escolaridade, sendo considerados com incapacidade cognitiva os indivíduos com resultado do Mini Exame do Estado Mental: $\leq 13$ pontos para analfabetos; $\leq 18$ para indivíduos com um a oito anos de escolaridade e $\leq 24$ pontos para indivíduos com mais de oito anos de escolaridade. Sendo assim, a variável cognição foi categorizada em "cognição preservada" e "suspeita de incapacidade cognitiva".

A funcionalidade dos pacientes foi avaliada por meio da Escala de Katz para Atividades Básicas de Vida Diária (AVD), de Katz et al..$^{28}$, adaptada para o Brasil por Lino et al. ${ }^{29}$, e da escala de Lawton e Brody ${ }^{30}$ para Atividades Instrumentais de Vida Diária (AIVD), analisada para o contexto brasileiro por Santos e Virtuoso-Junior ${ }^{31}$. A escala de Katz é constituída por seis itens, relacionados às atividades consideradas básicas (tomar banho, vestir-se, uso de vaso sanitário, transferência, continência e alimentação). Em cada item há uma classificação que indica "independência", "dependência parcial" ou "dependência total" para a realização das atividades básicas. A pontuação total varia de zero a seis pontos, sendo que "zero" indica total "independência para o desempenho de todas as atividades", a pontuação "seis" indica "dependência total na realização de todas as atividades" e a pontuação intermediária indi- 
ca a "dependência total ou parcial em quaisquer das atividades". Neste estudo, a variável AVD foi dicotomizada em "independente" para os idosos que obtiveram zero pontos na escala, e em "dependente parcial", para idosos que obtiveram pontuação maior que zero. A escala de Lawton e Brody ${ }^{30}$ consiste em sete questões, cada com três opções de resposta que apresentam peso de um a três pontos. Desse modo, a escala tem pontuação total que varia de 7 a 21 pontos. Neste estudo, a variável AIVD foi dicotomizada em "independente", para os idosos que obtiveram 21 pontos na escala, e em "dependente parcial", para idosos que obtiveram pontuação menor de 21 .

Os medicamentos presentes na prescrição médica apresentada foram classificados de acordo com Anatomical Therapeutic Chemical (ATC) ${ }^{32}$, nos $1^{\circ}$ e $5^{\circ}$ níveis (grupo anatômico principal e subgrupo químico, respectivamente). Empregou-se tal classificação para identificar o número de idosos que utilizavam simultaneamente três fármacos ativos no Sistema Nervoso Central (SNC). Segundo os critérios de Beers para uso de medicamentos potencialmente inapropriados em adultos idosos, o uso concomitante de três ou mais fármacos que atuam no SNC aumenta $o$ risco de quedas e fraturas ${ }^{33}$. Tais fármacos compreendem antipsicóticos, benzodiazepínicos, não benzodiazepínicos, hipnóticos agonistas dos receptores de benzodiazepínicos, antidepressivos tricíclicos, inibidores seletivos da recaptação de serotonina e opioides ${ }^{33}$. A classificação ATC também foi empregada para identificar a frequência dos fármacos utilizados por idosos expostos à polifarmácia e à polifarmácia excessiva.

A associação de cada variável explicativa qualitativa com as variáveis resposta foi investigada através do Teste Qui-Quadrado de Pearson ou do Teste Exato de Fisher. As variáveis significativas a $10 \%$ foram incluídas no modelo de regressão logística multivariado inicial. Permaneceram no modelo de regressão logística multivariado final as variáveis que continuaram significativas a $5 \%$. Para estas, foi estimada a razão de chances (RC), com intervalo de confiança de 95\% (IC95\%). A análise estatística foi realizada no software Statistical Package for Social Sciences (SPSS ${ }^{\circledR}$ ) versão $21^{34}$.

\section{Resultados}

Dos 234 idosos entrevistados, sete participantes não atenderam aos critérios de inclusão, resultando em 227 participantes.
Em relação às características sociodemográficas, este grupo apresentou predomínio de mulheres $(70,9 \%)$, com até 70 anos de idade $(50,2 \%)$, cor de pele preta/parda/indígena (58,6\%), escolaridade de até oito anos $(75,7 \%)$, sem companheiro $(61,6 \%)$, que não moram sozinhos $(76,2 \%)$ e com renda de até três salários mínimos $(60,9 \%)$. Quanto às características clínicas, pouco mais da metade $(50,2 \%)$ dos idosos apresentavam até três doenças. A autopercepção de saúde foi autorrelatada como positiva em $69,9 \%$ dos idosos e $77,5 \%$ não apresentaram sintomas de depressão. Em relação às características funcionais, a maioria dos idosos apresentou cognição preservada $(86,1 \%)$, dependência parcial para Atividades Instrumentais de Vida Diária $(77,1 \%)$ e independência para Atividades Básicas de Vida Diária (76,5\%). Nas características relacionadas à utilização de medicamentos, $26,9 \%$ apresentaram baixa adesão e $43,1 \%$ alta adesão. A polifarmácia foi encontrada para $57,7 \%$ dos idosos e a polifarmácia excessiva apenas $4,8 \%$ dos usuários. Somente $1,3 \%$ dos idosos utilizavam três ou mais fármacos que atuam no SNC (Tabela 1).

Os idosos entrevistados estavam em uso de 1 a 13 fármacos, com média de 5,2 e mediana de 5 Na Tabela 2 estão descritos os grupos de fármacos mais utilizados por idosos em polifarmácia e em polifarmácia excessiva, de acordo com a classificação anatômica da ATC (nível 1). A maior parte dos fármacos atuam sobre o sistema cardiovascular (polifarmácia - 49,2\%; polifarmácia excessiva - 37,3\%), seguidos dos que atuam sobre o trato alimentar e metabolismo (polifarmácia 19,0\%; polifarmácia excessiva - 28,0\%) e sistema nervoso (polifarmácia - 12,8\%; polifarmácia excessiva - 10,2\%). Considerando o nível 5 da ATC (fármaco) os fármacos mais utilizados por idosos em polifarmácia (Tabela 3), foram sinvastatina $(7,7 \%)$, hidroclorotiazida $(7,4 \%)$, losartana (7,0\%), ácido acetilsalicílico (6,3\%), anlodipino (5,9\%), enalapril (5,7\%) e omeprazol $(5,1 \%)$; nos idosos expostos à polifarmácia excessiva, os fármacos mais frequentes foram enalapril $(6,8 \%)$, omeprazol (6,8\%), hidroclorotiazida (5,7\%), ácido acetilsalicílico (5,7\%), anlodipino (5,7\%), metformina $(5,7 \%)$, insulina humana $(5,7 \%)$, glibenclamida $(4,5 \%)$ e sinvastatina $(4,5 \%)$.

A polifarmácia teve associação univariada significativa com as variáveis sociodemográficas idade $(p=0,053)$, sendo maior em idosos com até 70 anos de idade, e com a escolaridade $(\mathrm{p}=0,093)$, sendo mais prevalente em idosos com mais de oito anos de estudo. Todas as variáveis clínicas mostraram-se associadas à polifarmácia, 
Tabela 1. Características sociodemográficas, clínicas, funcionais e farmacoterápicas de 227 idosos atendidos em farmácias de duas Unidades Básicas de Saúde de Belo Horizonte-MG (2013-2014).

\begin{tabular}{|c|c|c|}
\hline Variáveis & $\mathbf{n}^{1}$ & $\%$ \\
\hline \multicolumn{3}{|l|}{ Sociodemográficas } \\
\hline \multicolumn{3}{|l|}{ Sexo } \\
\hline Feminino & 161 & 70,9 \\
\hline Masculino & 66 & 29,1 \\
\hline \multicolumn{3}{|l|}{ Idade } \\
\hline$\leq 70$ anos & 114 & 50,2 \\
\hline$>70$ anos & 113 & 49,8 \\
\hline \multicolumn{3}{|l|}{ Cor da pele } \\
\hline Branca ou amarela & 94 & 41,6 \\
\hline Preta, parda ou indígena & 132 & 58,4 \\
\hline \multicolumn{3}{|l|}{ Escolaridade } \\
\hline$\leq 8$ anos & 171 & 75,7 \\
\hline$>8$ anos & 55 & 24,3 \\
\hline \multicolumn{3}{|l|}{ Tem companheiro } \\
\hline Sim & 86 & 38,4 \\
\hline Não & 138 & 61,6 \\
\hline \multicolumn{3}{|l|}{ Mora sozinho (a) } \\
\hline Sim & 54 & 23,8 \\
\hline Não & 173 & 76,2 \\
\hline \multicolumn{3}{|l|}{$\operatorname{Renda}^{2}$} \\
\hline$\leq 3$ salários mínimos & 131 & 60,9 \\
\hline > 3 salários mínimos & 84 & 39,1 \\
\hline \multicolumn{3}{|l|}{ Clínicas } \\
\hline \multicolumn{3}{|l|}{ Número de doenças } \\
\hline$\leq 3$ & 114 & 50,2 \\
\hline$>3$ & 113 & 49,8 \\
\hline \multicolumn{3}{|l|}{ Autopercepção de saúde } \\
\hline Positiva & 158 & 69,9 \\
\hline Negativa & 68 & 30,1 \\
\hline \multicolumn{3}{|l|}{ Sintomas de depressão } \\
\hline Sim & 50 & 22,5 \\
\hline Não & 172 & 77,5 \\
\hline
\end{tabular}

que foi mais prevalente em idosos com mais de três doenças $(p=0,000)$, que apresentaram autopercepção de saúde negativa $(\mathrm{p}=0,05)$ e sintomas de depressão $(\mathrm{p}=0,020)$. As características de funcionalidade e farmacoterápicas não apresentaram associação significativa com polifarmácia (Tabela 4).

A polifarmácia excessiva mostrou-se mais prevalente entre idosos que apresentavam mais de três doenças $(p=0,005)$, com autopercepção de saúde negativa $(\mathrm{p}=0,073)$ e com dependência parcial nas Atividades Instrumentais de Vida Diária $(p=0,092)$. Estes resultados devem ser in-
Tabela 1. Características sociodemográficas, clínicas, funcionais e farmacoterápicas de 227 idosos atendidos em farmácias de duas Unidades Básicas de Saúde de Belo Horizonte-MG (2013-2014).

\begin{tabular}{|c|c|c|}
\hline \multirow{2}{*}{$\begin{array}{l}\text { Varıavels } \\
\text { Funcionalidade }\end{array}$} & $\mathbf{n}^{1}$ & $\%$ \\
\hline & & \\
\hline Cognição & & \\
\hline Preservada & 192 & 86,1 \\
\hline
\end{tabular}

Atividades Instrumentais de Vida

Diária

$\begin{array}{lrc}\text { Independente } & 52 & 22,9 \\ \text { Dependente parcial } & 175 & 77,1 \\ \text { Atividades Básicas de Vida Diária } & & \\ \text { Independente } & 173 & 76,6 \\ \text { Dependente parcial } & 53 & 23,4\end{array}$

Farmacoterápicas

Três ou mais fármacos $(\geq 3)$ que atuam no $\mathrm{SNC}^{3}$

$\begin{array}{lrr}\text { Sim } & 3 & 1,3 \\ \text { Não } & 224 & 98,7 \\ \text { Polifarmácia ( } \geq 5 \text { medicamentos) } & & \\ \text { Sim } & 131 & 57,7 \\ \text { Não } & 96 & 42,3\end{array}$

Polifarmácia excessiva $(\geq 10$

medicamentos)

\begin{tabular}{lrr} 
Sim & 11 & 4,8 \\
Não & 216 & 95,2 \\
\hline
\end{tabular}

${ }^{1}$ Total varia de acordo com a informação ignorada; ${ }^{2} \mathrm{O}$ valor do salário mínimo vigente era R\$ 724,00 (USD 227,8); ${ }^{3}$ Sistema Nervoso Central.

terpretados com cautela devido ao baixo número de idosos com polifarmácia excessiva (11 idosos).

As variáveis que apresentaram associação significativa (a 10\%) com polifarmácia foram incluídas no modelo de regressão logística multivariado, permanecendo no modelo final aquelas que continuaram significativas (a 5\%). No modelo final, as condições "ter idade menor ou igual a 70 anos" e "apresentar mais de três doenças" aumentam, respectivamente, em 1,93 e 4,24 vezes a chance do idoso estar em polifarmácia (Tabela 5).

O modelo de regressão logística multivariado para polifarmácia excessiva não pôde ser ajustado devido ao baixo número de observações do evento. 
Tabela 2. Medicamentos mais utilizados pelos idosos em polifarmácia ${ }^{1}$ e em polifarmácia excessiva ${ }^{2}$ atendidos em farmácias de duas Unidades Básicas de Saúde de Belo Horizonte-MG (2013-2014), segundo o nível 1 da classificação ATC $^{3}$.

\begin{tabular}{lccc}
\hline \multicolumn{1}{c}{ Grupo anatômico } & Código ATC & Polifarmácia & $\begin{array}{c}\text { Polifarmácia } \\
\text { excessiva }\end{array}$ \\
\cline { 3 - 4 } & (nível 1) & n (\%) & n (\%) \\
\hline Sistema cardiovascular & $\mathrm{C}$ & $432(49,2)$ & $44(37,3)$ \\
Trato alimentar e metabolismo & $\mathrm{A}$ & $167(19,0)$ & $33(28,0)$ \\
Sistema nervoso & $\mathrm{N}$ & $112(12,8)$ & $12(10,2)$ \\
Sangue e órgãos formadores de sangue & $\mathrm{B}$ & $75(8,5)$ & $8(6,8)$ \\
Sistema músculo-esquelético & $\mathrm{M}$ & $33(3,8)$ & $7(6,0)$ \\
Preparações hormonais sistêmicas, (exceto & $\mathrm{H}$ & $30(3,4)$ & $8(6,8)$ \\
hormônios sexuais e insulina) & & & $4(3,4)$ \\
Sistema respiratório & $\mathrm{R}$ & $15(1,7)$ & $2(1,7)$ \\
Anti-infecciosos para uso sistêmico & $\mathrm{J}$ & $8(0,9)$ & - \\
Dermatológicos & $\mathrm{D}$ & $3(0,3)$ & - \\
Órgãos sensoriais & $\mathrm{S}$ & $1(0,1)$ & - \\
Sistema genitourinário e hormônios sexuais & $\mathrm{G}$ & $877(100)$ & $118(100)$ \\
Total & & & - \\
\hline${ }^{1}$ Uso de cinco medicamento ou mais; ${ }^{2}$ Uso de dez medicamentos ou mais; ${ }^{3}$ Classificação Anatomical Therapeutic Chemical.
\end{tabular}

Tabela 3. Medicamentos mais utilizados pelos idosos em polifarmácia e polifarmácia excessiva atendidos em farmácias de duas Unidades Básicas de Saúde de Belo Horizonte-MG (2013-2014), segundo o nível 5 da classificação ATC.

\begin{tabular}{lccc}
\hline \multirow{2}{*}{ Substância química } & Código ATC (nível 5) & Polifarmácia & Polifarmácia excessiva \\
\cline { 3 - 4 } sinvastatina & C10AA01 & n (\%) & n (\%) \\
hidroclorotiazida & C03AA03 & $64(7,7)$ & $4(4,5)$ \\
losartana & C09CA01 & $62(7,4)$ & $5(5,7)$ \\
ácido acetilsalicílico & B01AC06 & $58(7,0)$ & $2(2,3)$ \\
anlodipino & C08CA01 & $53(6,3)$ & $5(5,7)$ \\
enalapril & C09AA02 & $49(5,9)$ & $5(5,7)$ \\
omeprazol & A02BC01 & $48(5,7)$ & $6(6,8)$ \\
atenolol & C07AB03 & $43(5,1)$ & $6(6,8)$ \\
metformina & A10BA02 & $40(4,8)$ & $1(1,1)$ \\
levotiroxina & H03AA01 & $38(4,5)$ & $5(5,7)$ \\
furosemida & $22(2,6)$ & $3(3,4)$ \\
glibenclamida & C03CA01 & $22(2,6)$ & $3(3,4)$ \\
paracetamol & A10BB01 & $19(2,3)$ & $4(4,5)$ \\
insulina humana & N02BE01 & $18(2,2)$ & $1(1,1)$ \\
clonazepam & A10AC01 & $17(2,0)$ & $5(5,7)$ \\
Outros & N03AE01 & $16(1,9)$ & $1(1,1)$ \\
Total & & $308(35,1)$ & $62(52,5)$ \\
\hline
\end{tabular}

\section{Discussão}

No presente estudo, foi possível identificar que a frequência de polifarmácia foi elevada entre os idosos da atenção primária e que esteve as- sociada positivamente com idade até 70 anos e ter mais de três doenças. Os resultados estão em concordância com a Pesquisa Nacional de Acesso, Utilização e Uso Racional de Medicamentos $(\text { PNAUM) })^{14,35} \mathrm{e}$ estudos realizados na Irlanda ${ }^{13} \mathrm{e}$ 
Tabela 4. Análise univariada da associação entre polifarmácia e polifarmácia excessiva e as características sociodemográficas, clínicas, funcionais e farmacoterápicas de 227 idosos atendidos em farmácias de duas Unidades Básicas de Saúde de Belo Horizonte-MG (2013-2014).

\begin{tabular}{|c|c|c|c|c|}
\hline \multirow{2}{*}{ Variáveis } & \multicolumn{2}{|c|}{ Polifarmácia } & \multicolumn{2}{|c|}{ Polifarmácia excessiva } \\
\hline & n (\%) & Valor-p $^{3}$ & n (\%) & Valor-p ${ }^{4}$ \\
\hline Geral $^{1}$ & $131(57,7)$ & - & $11(4,8)$ & - \\
\hline \multicolumn{5}{|l|}{ Sociodemográficas } \\
\hline Sexo & & 0,748 & & 1 \\
\hline Feminino & $94(58,4)$ & & $8(5,0)$ & \\
\hline Masculino & $37(56,1)$ & & $3(4,6)$ & \\
\hline Idade & & 0,053 & & 0,539 \\
\hline$\leq 70$ anos & $73(64,0)$ & & $7(6,1)$ & \\
\hline$>70$ anos & $58(51,3)$ & & $4(3,5)$ & \\
\hline Cor da pele & & 0,800 & & 0,368 \\
\hline Branca ou amarela & $55(58,5)$ & & $3(3,2)$ & \\
\hline Preta, parda ou indígena & $75(56,8)$ & & $8(6,1)$ & \\
\hline Escolaridade & & 0,093 & & 0,469 \\
\hline$\leq 8$ anos & $93(54,4)$ & & $7(4,1)$ & \\
\hline$>8$ anos & $37(67,3)$ & & $4(7,3)$ & \\
\hline Tem companheiro & & 0,762 & & 0,537 \\
\hline Sim & $51(59,3)$ & & $3(3,5)$ & \\
\hline Não & $79(57,2)$ & & $8(5,8)$ & \\
\hline Mora sozinho(a) & & 0,562 & & 0,726 \\
\hline Sim & $33(61,1)$ & & $3(5,6)$ & \\
\hline Não & $98(56,6)$ & & $8(4,6)$ & \\
\hline Renda & & 0,230 & & 1 \\
\hline$\leq 3$ salários mínimos & $81(61,8)$ & & $7(5,3)$ & \\
\hline > 3 salários mínimos & $45(53,6)$ & & $4(4,8)$ & \\
\hline \multicolumn{5}{|l|}{ Clínicas } \\
\hline Número de doenças & & 0,000 & & 0,005 \\
\hline$\leq 3$ & $47(41,2)$ & & $1(0,9)$ & \\
\hline$>3$ & $84(74,3)$ & & $10(8,9)$ & \\
\hline Autopercepção de saúde & & 0,005 & & 0,092 \\
\hline Positiva & $82(51,9)$ & & $5(3,2)$ & \\
\hline Negativa & $49(72,1)$ & & $6(8,8)$ & \\
\hline Sintomas de depressão & & 0,020 & & 0,273 \\
\hline Não & $92(53,5)$ & & $7(4,1)$ & \\
\hline Sim & $36(72,0)$ & & $4(8,0)$ & \\
\hline \multicolumn{5}{|l|}{ Funcionalidade } \\
\hline Cognição & & 0,209 & & 0,653 \\
\hline Preservada & $107(55,7)$ & & $9(4,7)$ & \\
\hline Suspeita de incapacidade cognitiva & $21(67,7)$ & & $2(6,5)$ & \\
\hline Atividades Instrumentais de Vida Diária & & 0,336 & & 0,073 \\
\hline Independente & $27(51,9)$ & & $0(0,0)$ & \\
\hline Dependente parcial & $104(59,4)$ & & $11(6,3)$ & \\
\hline Atividades Básicas de Vida Diária & & 0,819 & & 0,290 \\
\hline Independente & $101(58,4)$ & & $7(4,1)$ & \\
\hline Dependente parcial & $30(56,6)$ & & $4(7,6)$ & \\
\hline \multicolumn{5}{|l|}{ Farmacoterápicas } \\
\hline $\begin{array}{l}\text { Três ou mais fármacos }(\geq 3) \text { que atuam no } \\
\text { SNC }^{2}\end{array}$ & & 0,136 & & 1 \\
\hline Sim & $3(100)$ & & $0(0,0)$ & \\
\hline Não & $128(57,1)$ & & $11(4,9)$ & \\
\hline
\end{tabular}

${ }^{1}$ Total varia de acordo com a informação ignorada; ${ }^{2}$ SNC: Sistema Nervoso Central; ${ }^{3}$ Teste do Qui-quadrado; ${ }^{4}$ Teste Exato de Fisher. 
Tabela 5. Resultados dos modelos de regressão multivariados para polifarmácia em 227 idosos atendidos em farmácias de duas Unidades Básicas de Saúde de Belo Horizonte-MG (2013-2014).

\begin{tabular}{lcccccc}
\hline \multirow{2}{*}{\multicolumn{1}{c}{ Variável }} & \multicolumn{3}{c}{ Modelo inicial $^{1}$} & \multicolumn{3}{c}{ Modelo Final $^{2}$} \\
\cline { 2 - 7 } & RC & IC95\% & Valor-p & RC & IC95\% & Valor-p \\
\hline Idade $\leq 70$ anos & 1,61 & $0,89-2,93$ & 0,115 & 1,93 & $0,98-3,81$ & 0,053 \\
Escolaridade $>8$ anos & 1,88 & $0,93-3,80$ & 0,074 & - & - & - \\
Número de doenças $>3$ & 4,38 & $2,38-8,08$ & 0,000 & 4,24 & $2,39-7,51$ & 0,000 \\
Autopercepção de saúde negativa & 1,42 & $0,70-1,19$ & 0,330 & - & - & - \\
Com sintomas de depressão & 1,79 & $0,84-3,82$ & 0,125 & - & - & - \\
\hline
\end{tabular}

RC: razão de chances; IC: intervalo de confiança. Teste de qualidade de ajuste de Hosmer-Lemeshow: ${ }^{1} \mathrm{p}=0,438,{ }^{2} \mathrm{p}=0,929$.

Espanha ${ }^{36}$, com idosos atendidos na atenção primária à saúde. $\mathrm{O}$ estudo da PNAUM, que avaliou polifarmácia em maiores de 60 anos, encontrou prevalência de $60 \%$ entre idosos com quatro ou mais doenças ${ }^{35}$, reforçando a associação encontrada. Vale ressaltar que a heterogeneidade entre os estudos de polifarmácia quanto à faixa etária dos participantes (população total, maiores de $55,65,70$ ou 75 anos) e cenário de pesquisa (atenção primária, comunidade, instituições de longa permanência) dificulta a interpretação e a comparação dos resultados.

Até o presente momento, poucos estudos foram encontrados na literatura tendo a atenção primária como cenário para análise da prevalência de polifarmácia e de polifarmácia excessiva e seus fatores associados na população idosa. Além disso, não foram encontrados estudos que avaliaram as caraterísticas clínicas e de funcionalidade simultaneamente, como no presente estudo.

A prevalência de polifarmácia observada neste estudo foi superior àquelas encontradas em pesquisas de base populacional conduzidas no Brasil $3,5,14,35,37-39$; porém, todas mostram que o uso de múltiplos medicamentos é comum entre idosos.

Em relação à polifarmácia excessiva, a prevalência variou de $6 \%$ a $47 \%$ em estudos anteriores $^{10,13,40}$, acima do encontrado neste estudo. Cabe mencionar que os estudos supracitados são de base populacional, ou seja, utilizaram amostras maiores do que a deste trabalho, o que poderia explicar a polifarmácia excessiva aparentemente mais frequente. Outra questão que pode influenciar uma menor prevalência de polifarmácia excessiva no presente estudo, em relação a outros estudos, é que, neste trabalho, observou-se menor exposição à polifarmácia excessiva em pessoas com idade mais avançada ( $\geq 70$ anos). Nos demais estudos ${ }^{10,13,40}$, a prevalência de polifarmácia excessiva aumentou progressivamente com o avançar da idade. A variabilidade na prevalência observada entre os estudos se deve a variações nas práticas clínicas e nos comportamentos dos pacientes em diferentes países, bem como a diferenças na idade da amostra, na natureza da fonte de dados e nas unidades de análise $\mathrm{e}^{41,42}$.

Para Silveira et al. ${ }^{5}$ outro fator que pode contribuir para elevar o número de medicamentos utilizados por idosos é a prescrição distorcida ou impulsiva que ocorre em alguns casos, na tentativa de amenizar sintomas ou obter respostas mais rápidas em relação a medidas não farmacológicas. E, nesse contexto, pode-se dar início à cascata da prescrição, que ocorre quando os efeitos adversos dos medicamentos podem ser atribuídos de forma equivocada a uma condição médica e, dessa forma, um novo medicamento é prescrito para tratar um efeito adverso de outro medicamento $^{43}$. Essa conduta pode levar a diversas consequências, como o aumento do risco de Reações Adversas a Medicamentos (RAM), que eleva a morbimortalidade em idosos ${ }^{5,44-46}$, e o aumento do grau de fragilidade no idoso, que apresentou relação direta com polifarmácia em um estudo desenvolvido com adultos idosos franceses ${ }^{47}$.

Os fármacos mais utilizados por idosos em polifarmácia e em polifarmácia excessiva foram os que atuam sobre o sistema cardiovascular, resultado compatível com um estudo anterior, parte da PNAUM, também realizado na atenção primária à saúde ${ }^{14}$. Em outros estudos que analisaram o perfil de utilização de medicamentos por idosos em polifarmácia, os fármacos que atuam sobre o sangue e órgãos formadores de sangue foram os mais utilizados ${ }^{3,42}$.

Dentro de cada grupo anatômico, os fármacos e/ou sua frequência de uso variaram entre os idosos em polifarmácia e em polifarmácia excessiva. Entre os fármacos que atuam no sistema cardiovascular, sinvastatina, hidroclorotiazida e losartana foram os mais utilizados no grupo de 
polifarmácia, achado semelhante ao do estudo da PNAUM, que encontrou a sinvastatina e a losartana entre os fármacos mais utilizados pela população em polifarmácia ${ }^{14}$. A predominância de fármacos para uso crônico observada neste estudo demonstra que a polifarmácia pode ter relação com tratamentos longos, levando à necessidade de atenção e monitoramento continuados, por parte dos profissionais de saúde.

Em relação ao trato alimentar e metabolismo, o omeprazol foi mais prevalente no grupo de polifarmácia excessiva, figurando como o fármaco mais utilizado, juntamente com o enalapril. O consumo elevado do omeprazol, observado no presente estudo, pode ter relação com a inclusão desse fármaco ao tratamento do paciente, a fim de aliviar sintomas gástricos associados ao uso excessivo de medicamentos, ou seja, como consequência da polifarmácia ${ }^{5}$. Além disso, o uso do omeprazol e de outros fármacos que atuam reduzindo a acidez gástrica, de forma profilática e nem sempre racional, tem sido documentado, o que pode explicar sua alta prevalência de uso por idosos neste estudo ${ }^{3,14}$.

Idosos se queixam com frequência de insônia, ansiedade e estados de confusão mental, o que contribui para o aumento da prescrição de fármacos que atuam no Sistema Nervoso Central ${ }^{5}$. Entre os fármacos que atuam no sistema nervoso, o clonazepam foi o mais prevalente entre os idosos em polifarmácia e polifarmácia excessiva. $\mathrm{O}$ clonazepam é um benzodiazepínico de meia vida longa, incluído como inapropriado para idosos pelos Critérios de Beers e da Sociedade Americana de Geriatria ${ }^{43}$. O uso de benzodiazepínico aumenta significativamente o risco de quedas e fraturas por fragilidade nos idosos. Outras complicações descritas são declínio funcional, sonolência ou fadiga diurna, lentificação dos reflexos. O uso de benzodiazepínicos por idosos é justificado para desordens convulsivas, do sono REM (Rapid Eye Movement) e de ansiedade generalizada grave. Estratégias de desprescrição de benzodiazepínico devem ser implementadas visando evitar o uso prolongado e inapropriado por idosos ${ }^{44}$.

A associação significativa entre polifarmácia e idade corrobora a maioria dos estudos epidemiológicos ${ }^{3,5,8,35,39-42}$. Contudo, observou-se baixa prevalência de polifarmácia em idosos com 70 anos ou mais, semelhante ao encontrado em outros estudos brasileiros ${ }^{5,39}$ e em uma coorte retrospectiva conduzida na Itália ${ }^{42}$, o que reforça a associação encontrada com idade $\leq 70$ anos no presente estudo. É importante investigar as práticas medicamentosas em idosos com 80 anos ou mais, tendo em vista que esse grupo etário está sendo considerado distinto em relação às demais faixas etárias, devido ao aumento da expectativa de vida ${ }^{5}$.

A associação positiva de polifarmácia com múltiplas doenças é reconhecida, uma vez que os idosos que apresentam múltiplas comorbidades tendem a utilizar muitos medicamentos e, frequentemente, apresentam pior autopercepção de saúde ${ }^{5,8,14,42}$. Porém, nos idosos estudados a autopercepção de saúde negativa mostrou associação apenas na análise univariada.

É clara a necessidade de se ter um controle sobre as doenças crônicas por meio da elaboração de protocolos clínicos e diretrizes terapêuticas que contemplem ações de promoção da prescrição e do uso racional de medicamentos. Para isso, os tratamentos crônicos precisam ser monitorados desde a atenção primária, com ações voltadas para a prescrição e dispensação de medicamentos, levando a uma melhor compreensão do idoso, aumento as chances de sucesso do seu tratamento. É importante, ainda, que o conhecimento sobre multimorbidade e polifarmácia seja incorporado na prática médica, a fim de otimizar o controle das doenças crônicas, evitando a ocorrência de eventos adversos, prevenindo, assim, internações desnecessárias, incapacitação e perda da independência e da autonomia ${ }^{35}$.

A falta de uma definição de polifarmácia universalmente aceita é um desafio importante para discutir as consequências da polifarmácia relacionadas à segurança de medicamentos ${ }^{48}$. Além disso, na maioria das vezes, a polifarmácia é associada a desfechos clínicos negativos que comprometem a segurança e a qualidade de vida dos pacientes ${ }^{48}$. Dessa forma, as intervenções propostas no sentido de adequar a prescrição de pacientes expostos à polifarmácia, geralmente, são focadas apenas na redução do número de medicamentos prescritos ${ }^{48}$. A questão é que a polifarmácia nem sempre é um evento evitável ${ }^{39}$. As doenças crônicas altamente prevalentes (hipertensão arterial sistêmica, diabetes mellitus, etc.), em geral, são tratadas por meio da associação de fármacos. Portanto, o número específico de medicamentos utilizados não é por si só indicativo de adequação da terapia, pois o paciente pode estar em polifarmácia e todos os medicamentos podem ser clinicamente necessários e apropriados para o paciente. Nesse sentido, a revisão de esquemas terapêuticos múltiplos, especialmente em pacientes idosos com multimorbidade, deve ser incorporada na prática clínica de médicos generalistas e farmacêuticos, o que possibilita a avaliação da adequação dos medicamentos ${ }^{14}$. 
Mais recentemente, foram sugeridos os termos "potencialmente adequada" e "potencialmente problemática", a fim de diferenciar a polifarmácia apropriada da inapropriada. A polifarmácia é dita apropriada quando o uso dos medicamentos foi adequado ou foram prescritos considerando as evidências científicas, reconhecendo que os pacientes podem ser beneficiados com o uso de vários medicamentos. A polifarmácia é considerada problemática quando múltiplos medicamentos são prescritos de forma inapropriada ou o paciente não usufrui do benefício esperado de um medicamento. Esses conceitos buscam identificar prescrições potencialmente inapropriadas, principalmente em idosos, grupo de maior risco de eventos adversos ${ }^{48,49}$.

Os pontos fortes do presente estudo incluem a apresentação de prescrição médica no momento da entrevista, o que evita o viés de memória. Cabe citar que os instrumentos utilizados para avaliar as características clínicas e de funcionalidade são adaptados e validados para o Brasil, acrescentando valor metodológico ao estudo.

Uma limitação do estudo é ter incluído somente idosos que receberam pelo menos um medicamento na farmácia no momento da entrevista, podendo ter superestimado a prevalência de polifarmácia. Os medicamentos em uso fora da prescrição médica não foram considerados na coleta de dados, o que pode ter subestimado a frequência de polifarmácia. Outra limitação é não terem sido coletadas variáveis clínicas necessárias para a análise da adequação dos medicamentos e identificação dos idosos com polifarmácia apropriada, ampliando o conhecimento sobre esse problema de saúde publica. Apesar da relevância dos resultados, é importante cautela na sua generalização, pois o estudo foi desenvovido em dois centros de saúde de uma metrópole na região Sudeste do Brasil. Por fim, como o delineamento do presente estudo é transversal, não é possível estabelecer uma relação de causa e efeito. No entanto, foi demonstrado que há associação importante entre a utilização de medicamentos e as características dos indivíduos e, especificamente, das pessoas idosas.

$\mathrm{O}$ idoso, devido às características inerentes ao envelhecimento, exige um olhar e um cuidado diferenciados por parte dos profissionais de saúde, a fim de adotar a farmacoterapia mais apropriada e segura possível, respeitando as indicações, doses, interações e contraindicações de cada medicamento, e considerando critérios pré-definidos e as melhores evidências disponíveis. Nesse contexto, o farmacêutico tem um papel fundamental na equipe de saúde, pois é o profissional capaz de identificar problemas relacionados a medicamentos, realizar conciliação medicamentosa, revisar a farmacoterapia, podendo prevenir eventos adversos e agravos à saúde do idoso e, consequentemente, melhorando a qualidade de vida dessas pessoas.

O estudo mostrou que a polifarmácia é frequente entre os idosos nas UBS pesquisadas. Por isso, o uso de medicamentos por idosos com multimorbidade é uma questão importante que deve ser considerada na assistência ao idoso, sobretudo no âmbito da atenção primária à saúde. Nesse contexto, a polifarmácia figura como um indicador para se buscar o uso mais seguro e efetivo dos medicamentos, objetivando minimizar o risco de agravos à saúde. Com a população envelhecendo progressivamente, a tendência é aumentar o uso de medicamentos por idosos. Logo, o desenvolvimento de ações no sentido de garantir uma farmacoterapia adequada para a pessoa idosa, devem ser prioridade na agenda de planejamento do programa de atenção ao idoso e das ações de assistência farmacêutica do município de Belo Horizonte. As evidências atuais recomendam que intervenções para garantir a prescrição apropriada e segura para idosos compreendam medidas combinadas abrangendo estratégias de revisão da farmacoterapia, sistema informatizado de apoio a prescrição e dispensação, educação continuada para a equipe de saúde e serviços especializados em geriatria ${ }^{50}$.

Sendo assim, ressalta-se a necessidade da realização de mais pesquisas sobre polifarmácia apropriada na população idosa, sobretudo estudos de coorte, que visem avaliar o impacto da implantação do cuidado farmacêutico sobre a polifarmácia na atenção primária do SUS. A realização de estudos farmacoepidemiológicos prospectivos, abrangendo um número maior de UBS, são necessários para uma melhor caracterização da polifarmácia no município investigado, fornecendo elementos para otimizar a segurança e efetividade da farmacoterapia dos idosos.

\section{Conclusão}

A prevalência de polifarmácia obtida no presente estudo mostra que o uso de cinco ou mais medicamentos foi uma realidade entre os idosos atendidos em duas unidades básicas de saúde do SUS. Observou-se associação positiva entre polifarmácia e ter idade menor que 70 anos e apresentar mais de três doenças. Estes resultados fornecem dados importantes que podem orientar as políticas públicas relativas a utilização de medicamentos por idosos. 


\section{Colaboradores}

PC Oliveira trabalhou na análise dos dados, pesquisa bibliográfica e escrita do manuscrito. MR Silveira trabalhou na concepção e na redação final. MGB Ceccato trabalhou na coleta dos da- dos, na concepção e na redação final. AMM Reis trabalhou na coleta dos dados, na concepção e na redação. IVL Pinto trabalhou na coleta dos dados, na concepção e na redação. EA Reis trabalhou na análise dos dados e escrita do manuscrito.

\section{Referências}

1. Instituto Brasileiro de Geografia e Estatística (IBGE). Agência IBGE Notícias. Número de idosos cresce $18 \%$ em 5 anos e ultrapassa 30 milhões em 2017 [Internet]. [acessado 2018 Maio 22]. Disponível em: http://agenciadenoticias.ibge.gov.br/agencia-noticias/2012-agencia-de-noticias/noticias/20980-numero-de-idosos-cresce-18-em-5-anos-e-ultrapassa-30milhoes-em-2017.html.

2. Masnoon N, Shakib S, Kalisch-Ellett L, Caughey GE. What is polypharmacy? A systematic review of definitions. BMC Geriatr 2017; 17(1):230

3. Carvalho MFC, Romano-Lieber NS, Bergsten-Mendes G, Secoli SR, Ribeiro E, Lebrão ML. Polypharmacy among the elderly in the city of São Paulo, Brazil SABE study. Rev Bras Epidemiol 2012; 15(4):817-827.

4. Instituto para Práticas Seguras no Uso de Medicamentos (ISMP). Medicamentos potencialmente inadequados para idosos. Boletim ISMP 2017; 7(3):1-9.

5. Silveira EA, Dalastra L, Pagotto V. Polifarmácia, doenças crônicas e marcadores nutricionais em idosos. Rev Bras Epidemiol 2014; 17(4):818-829.

6. Kadam UT. Potential health impacts of multiple drug prescribing for older people: a case-control study. $J R$ Coll Gen Pract 2011; 61(583):128-130.

7. Mortazavi SS, Shati M, Keshtkar A, Malakouti SK, Basargan M, Assari S. Defining polypharmacy in the elderly: a systematic review protocol. BMJ Open 2016; 6(3):e010989.

8. Lim LM, McStea M, Chung WW, Azmi NN, Aziz SAA, Alwi S, Kamarulzaman A, Kamaruzzaman SB, Chua SS, Rajasuriar R. Prevalence, risk factors and health outcomes associated with polypharmacy among urban community-dwelling older adults in multiethnic Malaysia. Plos One 2017; 12(3):e173466.

9. Cooper JA, Cadogan CA, Patterson SM, Kerse N, Bradley MC, Ryan C, Hughes CM. Interventions to improve the appropriate use of polypharmacy in older people: a Cochrane systematic review. BMJ Open 2015; 5(12):e009235.

10. Jyrkkä J, Enlund H, Korhonen MJ, Sulkava R, Hartikainen S. Patterns of drug use and factors associated with polypharmacy and excessive polypharmacy in elderly persons: results of the Kuopio 75+ study: a cross-sectional analysis. Drugs Aging 2009; 26(6):493503.

11. Guthrie B, Makubate B, Hernandez-Santiago V, Dreischulte $\mathrm{T}$. The rising tide of polypharmacy and drugdrug interactions: population database analysis 19952010. BMC Med 2015; 13:74.
12. Hovstadius B, Petersson G. Factors leading to excessive polypharmacy. Clin Geriatr Med 2012; 28(2):159172.

13. Moriarty F, Hardy C, Bennett K, Smith SM, Fahey T. Trends and interaction of polypharmacy and potentially inappropriate prescribing in primary care over 15 years in Ireland: a repeated cross-sectional study. BMJ Open 2015; 5:9.

14. Nascimento RCRM, Álvares J, Guerra Junior AA, Gomes IC, Silveira MR, Costa EA, Leite SN, Costa KS, Soreiro OM, Guibu IA, Karnikowski MGO, Acurcio FA. Polifarmácia: uma realidade na atenção primária do Sistema Único de Saúde. Rev Saude Publica 2017; 51(Supl. 2):19s.

15. Pinto IVL, Reis AMM, Almeida-Brasil CC, Silveira MR, Lima MG, Ceccato MGB. Avaliação da compreensão da farmacoterapia entre idosos atendidos na Atenção Primária à Saúde de Belo Horizonte, MG, Brasil. Cien Saude Colet 2016; 21(11):3469-3481.

16. Belo Horizonte. Prefeitura Municipal. Censo BH Vida [Internet]. [acessado 2013 Abr 11 e Set 10]. Disponível em: http://pote.pbh/bhvida_novo/gestao/cadastrofamiliar/relatorio.php.

17. Belo Horizonte. Prefeitura Municipal. Secretaria Municipal de Saúde. Apresenta informações sobre a Atenção Primária e a Assistência Farmacêutica no Município de Belo Horizonte [Internet]. [acessado 2016 Out 10]. Disponível em: http://portalpbh.pbh.gov.br/pbh/ ecp/comunidade.do?app=saude.

18. Dean AG, Sullivan KM, Soe MM. Open Epi: Open Source Epidemiologic Statistics for Public Health, Version 3.01 [Internet]. [acessado 2013 Maio 15]. Disponível em: http://www.openepi.com/v37/Menu/OE_ Menu.htm.

19. Harrison C, Britt H, Miller G, Henderson J. Examining different measures of multimorbidity, using a large prospective cross-sectional study in Australian general practice. BMJ Open 2014; 4:e004694.

20. World Health Organization (WHO). The Whoqol Group. Development of the World Health Organization WHOQOL-B: quality of life assessment. Psychol Med 1998; 28:551-558.

21. Fleck MPA, Louzada S, Xavier M, Chachamovich E, Vieira G, Santos S, Pinzon V. Aplicação da versão em português do instrumento abreviado de avaliação da qualidade de vida "WHOQOL-bref”. Rev Saude Publica $2000 ; 34(2): 178-183$. 
22. Yesavage JA, Brink TL, Rose TL, Lum O, Huang V, Adey M, Leirer VO. Development and validation of a geriatric depression screening scale: a preliminary report. J Psychiatr Res 1983; 17(1):37-42.

23. Almeida OP, Almeida SA. Confiabilidade da versão brasileira da escala de depressão em geriatria (GDS) versão reduzida. Arq Neuropsiquiatr 1999; 57(2B):421-426.

24. Paradela EM, Lourenço RA, Veras RP. Validação da escala de depressão geriátrica em um ambulatório geral. Rev Saude Publica 2005; 39(6):918-923.

25. Folstein MF, Folstein SE, Mchugh PR. "Mini-Mental State." A practical method for grading the cognitive state of patients for the clinician. J Psychiatr Res 1975; 12(3):189-198.

26. Bertolucci PH, Brucki SMD, Campacci SR, Juliano Y. O mini-exame do estado mental em uma população geral: impacto da escolaridade. Arq Neuropsiquiatr 1994; 52:1-7.

27. Lourenço RA, Veras RP. Mini-Exame do Estado Mental: características psicométricas em idosos ambulatoriais. Rev Saude Publica 2006; 40(4):712-719.

28. Katz S, Ford AB, Moskowitz RW, Jackson BA, Jaffe MW. Studies of illness in the aged. The index of ADL: a standardized measure of biological and psychosocial function. JAMA 1963; 185(12):914-919.

29. Lino VTS, Pereira SRM, Camacho LAB, Ribeiro Filho ST, Buksman S,. Adaptação transcultural da Escala de Independência em Atividades da Vida Diária (Escala de Katz). Cad Saude Publica 2008; 24(1):103-112.

30. Lawton MP, Brody EM. Assessment of Older People: Self-maintaining and Instrumental Activities of Daily Living. Gerontologist 1969; 9(3):179-186.

31. Santos RL, Virtuoso-Junior JS. Confiabilidade da versão brasileira da escala de atividades de vida diária. RBPS 2008; 21(4):290-296.

32. World Health Organization (WHO). ATC/DDD Index 2014 [Internet]. [acessado 2014 Mar 1]. Disponível em: http://www.whocc.no/atc_ddd_index/.

33. American Geriatrics Society. American Geriatrics Society 2015 Updated Beers Criteria for Potentially Inappropriate Medication Use in Older Adults. J Am Geriatr Soc 2015; 63(11):2227-2246.

34. IBM Corp. Released 2012. IBM SPSS Statistics for Windows, Version 24.0. Armonk: IBM Corp; 2012.

35. Ramos LR, Tavares NUL, Bertoldi AD, Farias MR, Oliveira MA, Luiza VL, Dal Pizzol TS, Arrais PSD, Mengue SS. Polifarmácia e polimorbidade em idosos no Brasil: um desafio em saúde pública. Rev Saude Publica 2016; 50(Supl. 2):9s.

36. López TM, Camacho MOC, Morgado DP, Rubio SL, Camacho JCD, Serna JCM. Prevalencia de polimedicación y riesgo vascular en La población mayor de 65 años. Aten Primaria 2012; 44(4):216-222.

37. Loyola Filho AI, Uchoa E, Lima-Costa MF. A population-based study on use of medication by the elderly in Greater Metropolitan Belo Horizonte, Minas Gerais, Brazil. Cad Saude Publica 2006; 22(12):26572667.

38. Loyola Filho AI, Uchoa E, Firmo JOA, Lima-Costa MF. Influence of income on the association between cognitive impairment and polypharmacy: Bambuí Project. Rev Saude Publica 2008; 42(1):89-99.
39. Rozenfeld S, Fonseca MJM, Acurcio FA. Drug utilization and polypharmacy among the elderly: a survey in Rio de Janeiro City, Brazil. Rev Panam Salud Publica 2008; 23(1):34-43.

40. Morin L, Vetrano DL, Rizzuto D, Calderón-Larrañaga A, Fastbom J, Johnell K. Choosing wisely? Measuring the burden of medications in older adults near the end of Life: Nationwide, Longitudinal Cohort Study. Am J Med 2017; 130(8):927-936.

41. Linjakumpu T, Hartikainen S, Klaukka T, Veijola J, Kivelä S-L, Isoaho R. Use of medications and polypharmacy are increasing among the elderly. J Clin Epidemiol 2002; 55(8):809-817.

42. Slabaugh SL, Maio V, Templin M, Abouzaid S. Prevalence and risk of polypharmacy among the elderly in an outpatient setting. A retrospective cohort study in the Emilia-Romagna Region, Italy. Drugs Aging 2010; 27(12):1019-1028.

43. The 2019 American Geriatrics Society Beers Criteria ${ }^{\circledR}$ Update Expert Panel. American Geriatrics Society updated AGS Beers Criteria ${ }^{\circledR}$ for potentially inappropriate medication use in older adults. J Am Geriatr Soc 2019; 67(4):674-694.

44. Ng BJ, Counter DGL, Hilmer SN. Deprescribing benzodiazepines in older patients: impact of interventions targeting physicians, pharmacists, and patients. Drugs Aging 2018; 35(6):493-521.

45. Merel SE, Paauw DS. Common drug side effects and drug-drug interactions in elderly adults in primary care. J Am Geriatr Soc 2017; 65(7):1578-1585.

46. Secoli SR. Polifarmácia: interações e reações adversas no uso de medicamentos por idosos. Rev Bras Enferm 2010; 63(1):136-140.

47. Heer M, Robine J-M, Pinot J, Arvieu JJ, Ankri J. Polypharmacy and frailty: prevalence, relationship, and impact on mortality in a French sample of 2350 old people. Pharmacoepidemiol Drug Saf 2015; 24(6):637646.

48. Cadogan CA, Ryan C, Hughes CM. Appropriate polypharmacy and medicine safety: When many is not too many. Drug Saf 2016; 39(2):109-116.

49. Molokhia M, Majeed A. Current and future perspectives on the management of polypharmacy. BMC Fam Prac 2017; 18(1):70.

50. Santos NS, Marengo LL, Moraes FS, Barberato-Filho S. Interventions to reduce the prescription of inappropriate medicines in older patients. Rev Saúde Pública 2019; 53:7.

Artigo apresentado em 22/06/2018

Aprovado em 01/07/2019

Versão final apresentada em 03/07/2019

Editores-chefes: Romeu Gomes, Antônio Augusto Moura da Silva 